\title{
Primary hydatid cyst of anterior thigh - an unusual location
}

\author{
Suvendu Maji ${ }^{1}$, Makhan Lal Saha ${ }^{2}$ \\ 1 Chittaranjan National Cancer Institute, Kolkata, India. \\ 2 Institute of Post graduate medical education and research, Kolkata, India.
}

Key words: Hydatid cyst; intramuscular; zoonosis; thigh

\section{Introduction}

Hydatid disease is a zoonotic infection and is endemic in India. The most commonly affected organs are liver (75\%) followed by lungs (25\%).Primary skeletal muscle hydatidosis is extremely rare and occur in approximately in $2.3 \%$ of cases. Given its rarity and its close similarity with other entities like soft tissue tumour, abscess, sarcoma, diagnosis of intramuscular hydatid cyst may be challenging.

\section{Case presentation}

A 45 year old gentleman, security guard by profession presented to us with swelling of his left thigh for the last 3 months. The swelling was progressively increasing in size and was also associated with low grade intermittent fever for the last one month. There was no history of trauma to thigh or history of similar swelling elsewhere in body. He denied any difficulty in movement or pain in his thigh.

There was no other systemic complaints. He had no history of contacts with pets, animals or birds. His general survey was essentially normal. On local examination there was a nontender firm, mass with smooth surface and indistinct margin measuring approximately $20 \times 19 \mathrm{cms}$, occupying the entire anterior and lateral aspect of the left thigh. There were no palpable inguinal nodes.

A bedside ultrasonography done showed presence of multiple cystic lesions in the anterior aspect of his Magnetic Resonance Imaging (MRI) of left thigh showed a large peripherally enhancing multiloculated cystic mass of $111 \times 175 \times 133 \mathrm{~mm}$ located within the adductor group of muscles, along with displacement of vastii medialis and intermedius muscle (Figure 1 \&2). The bone marrow was normal. Chest $\mathrm{X}$ ray and USG abdomen did not reveal any other sites for hydatid cyst. Routine laboratory tests were normal. However serology for hydatid cyst was positive. He

Correspondence: Suvendu Maji

E-mail: drsuvendumaji@rediffmail.com

Received: 06-09-2017 Accepted: 19-10-2017

(iD) http://orcid.org/0000-0003-3815-7364

DOI: http://doi.org/10.4038/sljs.v35i3.8417
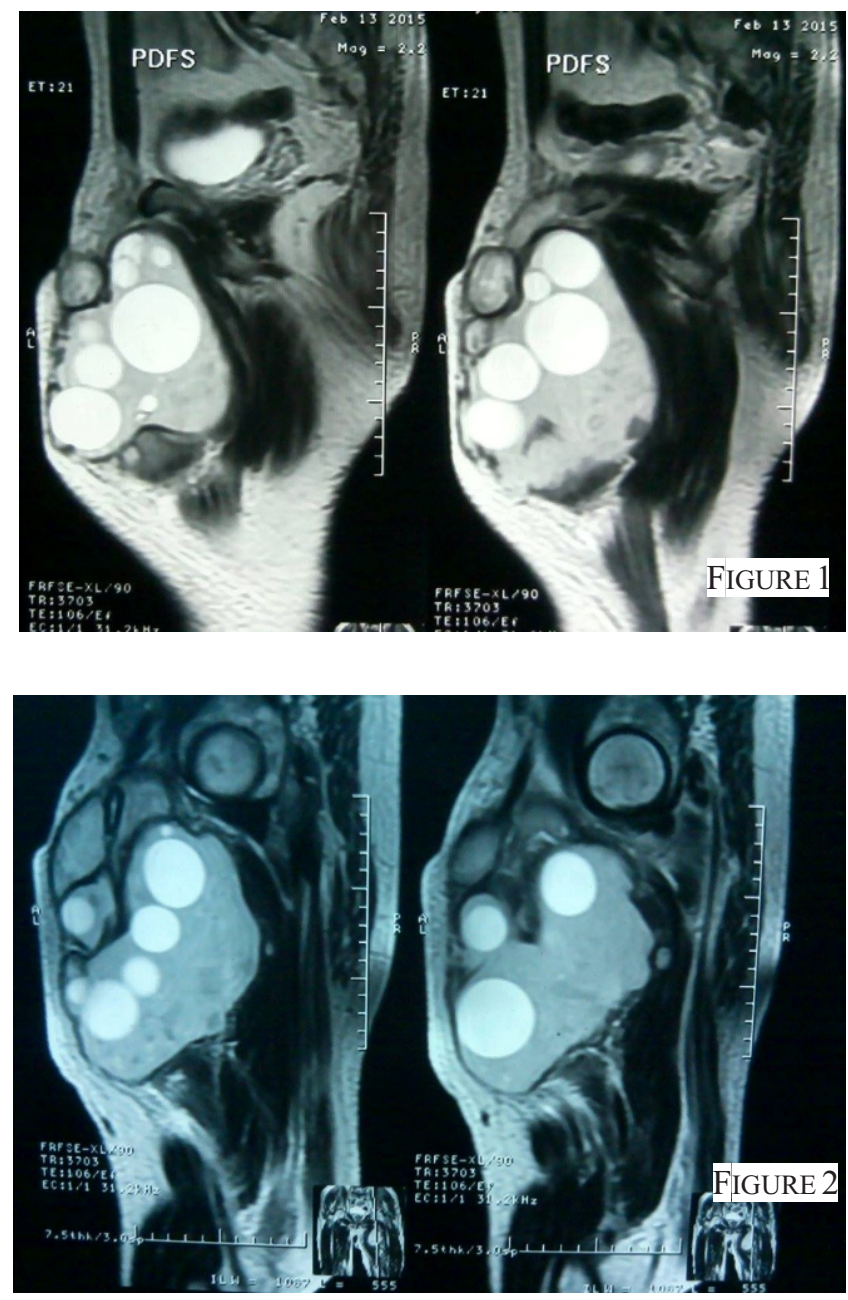

Figure $1 \& 2$. MRI of left thigh showed a large peripherally enhancing multiloculated cystic mass of $(111 \times 175 \times 133) \mathrm{mm}$ located within the adductor group of muscles,along with displacement of vastii medialis and intermedius muscle.

was given a course of antibiotic and antihelminthic treatment. The final histopathology report was 'infected hydatid cyst'. However the swelling did not subside. He was next planned for enbloc excision of the lump. Under regional anaesthesia the cyst was excised enbloc from the quadriceps muscle using an elliptical transverse incision. The cavity was irrigated with solicidal agents and closed. Postoperative recovery was uneventful. He continues to be in excellent state of health on 2 years follow up. 


\section{Discussion}

Hydatid cyst is caused by echinococcus granulosus where dog is the definitive host and man is often the accidental host. Lung and liver are the two most commonly affected organs. Intramuscular hydatid cyst can be either primary or occur secondarily due to spread from other involved organs [1]. Primary intramuscular hydatid cyst is extremely rare because contractility of muscle and presence of lactic acid often inhibit their growth $[2,3]$. Such cysts grow slowly as the incubation period ranges from 5-20 years.

Hence presentation may be several years after primary exposure, when most patients deny any history of exposure to pets or animals as was in our case. Diagnosis may be confirmed by a battery of laboratory tests and radiological investigations. The Casoni skin test is rarely used now. There are other serological tests out of which Immunoelectrophoresis is the most specific method.

Plain X rays, Ultrasonography, Computed tomography scan, MRI all has been used for diagnosis of muscular hydatid cyst [4]. MRI has been found to be superior to US and CT scan. It can delineate the exact relation between the cyst and the muscle group. The Rim Sign found on T2 weighted images of MRI has been described as a characteristic sign of Intramuscular Hydatid cyst, which is not a usual finding in cysts present elsewhere in the body [5]. Preoperative diagnosis is extremely important to avoid inadvertent rupture of the cyst and life threatening anaphlacic shock. Treatment incudes complete surgical excision of the cyst along with irrigation of the cavity with scolicidal agents. Systemic antihelminthic therapy may be given and the patients' needs to be kept in lifelong follow up for recurrences $[6,7]$.

\section{Conclusion}

In conclusion though hydatid cyst is a common disease its uncommon presentation as was in our case should be borne in mind. A combination of patients' history, clinical examination, serology and radiological findings should be used in arriving at diagnosis.

\section{References}

1. Jarboui S, Hlel A, Daghfous A, Bakkey MA, Sboui I. Unusual Location of Primary Hydatid Cyst: Soft Tissue Mass in the Supraclavicular Region of the Neck. Case Reports in Medicine. 2012;2012:484638.

DOI: http://dx.doi.org/10.1155/2012/484638.

2. Koc Z, Agildere AM, Yalcin O, et al. Primary hydatid cyst in the anterior thigh: sonographic findings. J Clin Ultrasound. 2004; 32(7):358-60.

DOI: http://dx.doi.org/10.1002/jcu.20044

3. Coexistence in unusual locations of hydatid cysts: thigh, breast and lung involvement Uysal, Mustafa et al.International Journal of Infectious Diseases, Volume 11 , Issue 5, 470 - 472 DOI: http://dx.doi.org/10.1016/j.ijid.2007.01.004

4. Rodrigues, G. Primary Subcutaneous Hydatid Cyst of the Thigh An Unusual Site With a Diagnostic Dilemma. Journal of Cutaneous Medicine and Surgery. 2015;20(3): 266-268. DOI: https://doi.org/10.1177/1203475415623778

5. Comert RB, Aydingoz U, Ucaner A, et al. Waterlily sign on MR imaging of primary intramuscular hydatidosis of sartorius muscle. Skeletal Radiol. 2003; 32: 420-3.

DOI: https://doi.org/10.1007/s00256-003-0661-x

6. Lamine A, Fikry T, Zryouil B: L'hydatidose primitive des muscles peripheriques a propos de $7 \mathrm{cas}$ (in French). Acta Orthop Belg 1993; 59:184-188

PMid:8372655

7. Marwah S., Subramanian P., Marwah N., Rattan K.N., Karwasra R.K. 2005. Infected primary intramuscular echinococcosis of thigh. Indian Journal of Pediatrics, 72, 799ñ800.

DOI: https://doi.org/10.1007/BF02734157

\section{Learning Points:}

- The diagnosis of a hydatid cyst should be kept in mind while evaluating a thigh lump.

- Detailed clinical history and high index of suspicion is key to diagnosis. 